\title{
Infarto agudo de miocardio manifestado como bloqueo de rama izquierda nuevo: revisión de la literatura
}

Fecha de recibido: 22 de agosto de 2017

Fecha de aprobación: 22 de enero de 2018
Forma de citar este artículo: Zuluaga $M$, Estrada AF, Caicedo J. Infarto agudo de miocardio manifestado como bloqueo de rama izquierda nuevo: revisión de la literatura. Med U.P.B. 2018;37(2):142-148. DOI:10.18566/medupb.v37n2.a08

1. Médico General, Cuidados Especiales Adulto, Hospital Pablo Tobón Uribe. Medellín, Colombia.

2. Especialista en Medicina de Urgencias, Universidad CES. Hospital Pablo Tobón Uribe. Medellín, Colombia

Dirección de correspondencia: Mateo Zuluaga Gómez. Correo electrónico: mateozg92@ hotmail.com

\section{Acute myocardial infarction manifested as new left bundle branch block: A literature review / Infarto agudo de miocardio manifestado como bloqueio do ramo esquerdo novo: revisão da literatura}

Mateo Zuluaga Gómez¹, Andrés Felipe Estrada Atehortúa², Johanna Caicedo Valle².

\section{RESUMEN}

A través del tiempo se ha hablado del hallazgo de bloqueo de rama izquierda del Haz de His como un posible equivalente ST, en el paciente que se presenta a los servicios médicos con dolor torácico. Es claro que en condiciones puntuales como inestabilidad hemodinámica o choque cardiogénico se cumple dicha premisa y el paciente debe ser manejado de manera invasiva urgente, sin embargo, hay otras condiciones que ameritan trabajo de campo adicional para abordar estos pacientes.

Palabras clave: choque cardiogénico; falla cardiaca; dolor torácico.

\section{ABSTRACT}

Over time, there has been discussion regarding the finding of left bundle branch block in the bundle of His as a possible ST-segment equivalent, in patients seeking medical attention with chest pain. It is clear that, in particular conditions such as hemodynamic instability or cardiogenic shock, this premise is met and patients must undergo emergency invasive procedures. However, there are other conditions that warrant further field work to treat these patients.

Keywords: cardiogenic shock; heart failure; chest pain.

\section{RESUMO}

A través do tempo se há falado da descoberta de bloqueio do ramo esquerdo de Feixe de His como um possível equivalente ST, no paciente que se apresenta aos serviços médicos com dor torácico. É claro que em condições pontuais como instabilidade hemodinâmica ou choque cardiogênico se cumpre dita premissa e o paciente deve ser manejado de maneira invasiva urgente, embora, há outras condições que merecem trabalho de campo adicional para abordar estes pacientes.

Palavras chave: choque cardiogênico; falha cardíaca; dor torácico.

\section{INTRODUCCIÓN}

La prevalencia del bloqueo de rama izquierda del Haz de His (BRIHH) en la población que presenta sintomatología compatible con síndrome coronario agudo es alrededor del 2-4\%. La mayoría de personas que lo presentan son de edad avanzada, mujeres y tienen antecedentes de enfermedad cardiovascular como hipertensión arterial y falla cardiaca, reflejando que además de tener un sustrato isquémico, también puede haber un componente de 
alteración en la estructura cardiaca ${ }^{1,2}$. Todo esto hace que dichos pacientes tengan mayor retraso en el diagnóstico y tratamiento adecuado (terapia de reperfusión) ${ }^{3}$, con el consecuente aumento en la morbimortalidad y empeoramiento en su pronóstico ${ }^{4,5}$. Dado que la presencia de bloqueo de rama izquierda es más prevalente en la población de edad avanzada y se asocia con patologías crónicas de base, es posible que su hallazgo exprese más un riesgo cardiovascular global a que sea solamente un indicador independiente de mortalidad ${ }^{6-8}$.

\section{EPIDEMIOLOGÍA}

\section{Manifestaciones electrocardiográficas}

Históricamente se ha asociado la presencia de infarto agudo de miocardio con el hallazgo de bloqueo de rama izquierda nuevo en el trazado electrocardiográfico de los pacientes que se presentan a un servicio médico con síntomas sugestivos de evento coronario agudo. Sin embargo, la evidencia apunta a que la mayoría de los pacientes con dicho hallazgo no cursan con un infarto ${ }^{9,10}$. Como se observó en la revisión realizada por Nilay Mehta y colaboradores, entre 69 pacientes con diagnóstico de bloqueo de rama izquierda nuevo, el $54 \%$ fue llevado a intervencionismo percutáneo temprano y solo el $22 \%$ tuvo evidencia de oclusión de un vaso epicárdico. Adicionalmente, el $28 \%$ presentó elevación de troponina I, sugiriendo que no todo bloqueo de rama izquierda se asocia con lesión isquémica del miocardio ${ }^{11}$. Tampoco se puede perder de vista que además de la enfermedad isquémica cardiaca, hay otras causas extracardiacas de liberación de troponinas al torrente sanguíneo, y si se tienen como único parámetro para el diagnóstico de infarto agudo de miocardio, se podría sobreestimar su prevalencia ${ }^{12}$. Estos hallazgos son reforzados por el trabajo de Larson y colaboradores, donde se realizó angiografía urgente a un grupo de 36 pacientes con bloqueo de rama izquierda nuevo o presumiblemente nuevo y se encontró que el $44 \%$ de ellos no tenía hallazgos patológicos a nivel coronario que explicaran su sintomatología al ingreso ${ }^{13}$. Todo esto sugiere que la presencia de un bloqueo de rama izquierdo nuevo en un paciente con dolor torácico agudo tiene un valor diagnóstico limitado, al identificar pacientes con oclusión coronaria aguda, y obliga a encontrar otras herramientas que ayuden a predecirla ${ }^{4}$.

En 1996, Sgarbossa et al, basados en información arrojada por el estudio GUSTO-1, analizaron diferentes patrones electrocardiográficos posiblemente relacionados con la presencia de infarto agudo de miocardio en el escenario de bloqueo de rama izquierda. De los datos encontrados, concluyeron que la elevación del ST mayor a $1 \mathrm{~mm}$ concordante con el complejo QRS en cualquier derivada (OR 25.2 con un IC 95\%,11.6-54.7), depresión del segmento ST mayor a 1mm en V1, V2 y V3 (OR 6 con un IC 95\%, 1.9-19.3) y elevación del segmento ST mayor a $5 \mathrm{~mm}$ discordante con el QRS (4.3 con un IC $95 \%, 1.8-10.6)$ se asociaron con la evento coronario agudo. Combinados, arrojan una sensibilidad de $78 \%$ y una especificidad del $90 \%$. Es así como a cada ítem se le asignó un puntaje, al primero 5 puntos, al segundo 3 puntos y al último 2 puntos; esto para aportar que un puntaje mayor o igual a 3 es altamente sugestivo de oclusión coronaria aguda $^{14,15}$. Estos hallazgos fueron validados por Hussam en un estudio con 267 pacientes que presentaron bloqueo de rama izquierdo e infarto agudo de miocardio, el cual reveló que un puntaje mayor a 3 se asociaba con la oclusión coronaria aguda. Además, se vio que la mortalidad a 30 días y un año fue de $13.5 \%$ y $25.2 \%$ respectivamente, con un importante aumento de la incidencia de shock cardiogénico y falla cardiaca. Esto sugiere que aquellos pacientes con un puntaje mayor a 3 son los que más se benefician de reperfusión temprana como la fibrinolisis ${ }^{16}$. Hay que tener presente que los pacientes que ingresan a urgencias con dolor en tórax de características anginosas y cuyo trazado electrocardiográfico muestra bloqueo de rama izquierda que no cumple los criterios mencionados, se benefician de monitoreo cardiaco continuo o de toma seriada de electrocardiogramas. En gran parte de los pacientes que realmente tienen oclusión coronaria en curso como etiología de su presentación clínica, posiblemente se aprecien cambios secuenciales o dinámicos en dichos registros, que apoyan de esta forma el diagnóstico de infarto agudo de miocardio ${ }^{17,18}$.

\section{Manifestaciones clínicas}

Algunas revisiones arrojan información que favorece la asociación entre bloqueo de rama izquierdo nuevo e IAM ST, como encontraron en una serie de 5742 pacientes con infarto agudo de miocardio. El 1.7\% (98 pacientes) tenía registro electrocardiográfico de bloqueo de rama izquierdo, donde más del 95\% de los pacientes que padecía bloqueo nuevo asociado con síntomas isquémicos, y que fueron llevados a arteriografía coronaria, presentaron evidencia de compromiso coronario. Adicionalmente, los pacientes que cumplieron el criterio de Sgarbossa, elevación del ST mayor a $1 \mathrm{~mm}$ concordante con el QRS, tuvieron menos tiempo entre el diagnóstico y la realización de intervencionismo percutáneo que los que no lo cumplieron. El 13.3\% de los pacientes con bloqueo de rama izquierdo presentaron marcadores negativos para daño cardiaco, $14.3 \%$ no experimentaron lesión coronaria en la angiografía que explicaran los síntomas y el 1\% no tuvo enfermedad coronaria significativa ${ }^{19,20}$. 
También se ha buscado establecer una asociación entre la presencia de síntomas anginosos en el contexto de un bloqueo de rama izquierdo, presumiblemente nuevo, y el curso de un infarto agudo de miocardio con elevación del segmento ST; sin embargo, los datos arrojados por pequeñas series de casos son inconcluyentes ${ }^{21,22}$. En una revisión de 69 pacientes con bloqueo de rama izquierdo nuevo, los síntomas más frecuentes de presentación en el servicio de urgencias fueron: dolor torácico (46.4\%), disnea $(21.7 \%)$ y paro cardiorrespiratorio (5.8\%), prediciendo la necesidad de revascularización coronaria con un LR de 1.9. Interesantemente, de estos 69 pacientes, solamente un $8.6 \%$ cumplió con criterios de Sgarbossa para infarto agudo de miocardio ${ }^{11,23}$.

\section{FISIOPATOLOGÍA}

El sistema de conducción eléctrico ventricular está compuesto por el Haz de His y sus ramas; una derecha, que es delgada y de corta trayectoria, y una izquierda que a su vez se subdivide en un fascículo anterosuperior y otro posteroinferior, más gruesos y extensos ${ }^{24}$. Cuando se produce un bloqueo en dicho sistema de conducción, la explicación puede ser tanto una única lesión localizada a nivel proximal de la vía (justo antes de la división de la rama izquierda en sus dos fascículos) o una gran lesión que compromete una mayor masa ventricular ${ }^{25}$. Cuando la lesión es isquémica, la mayoría de las ocasiones es secundaria a compromiso de la circulación de la pared anterior del ventrículo izquierdo. Cuando se compromete el tronco izquierdo o la parte proximal de la arteria descendente anterior, se afecta el tercio proximal del sistema de conducción, resultando en un infarto transmural que se ve como un bloqueo de rama derecha con bloqueo de fascículo anterosuperior izquierdo y no como un bloqueo de rama izquierda completo ${ }^{26}$. Cualquier proceso fisiopatológico que lleve a cambios en la estructura miocárdica, por ejemplo, hipertensión arterial crónica, falla cardiaca, cardiopatía isquémica o valvulopatía, puede producir bloqueo de rama del Haz de His, por ende, la mayoría de estos últimos bloqueos se dan de forma crónica con curso asintomático, por lo que es difícil determinar su antigüedad $^{27-29}$. Los pacientes con estas condiciones cardiacas se presentan con síntomas que pueden ser malinterpretados como equivalentes de síndrome coronario agudo. Sin embargo, es importante tener en cuenta que un bloqueo de rama izquierda secundario a infarto agudo de miocardio probablemente comprometa el estado hemodinámico, dado que se requiere una oclusión coronaria muy proximal para comprometer las ramas perforantes septales que irrigan la rama izquierda del $\mathrm{Haz}$ de $\mathrm{His}^{30,31}$.

\section{ENFOQUE DIAGNÓSTICO Y MANEJO}

La diferenciación de un infarto agudo de miocardio con elevación del segmento ST cobra vital importancia en cuanto a que la terapia urgente, en las primeras 12 horas de iniciados los síntomas, es sin duda la terapia de reperfusión ${ }^{32,33}$, ya sea tratamiento intervencionista temprano (revascularización coronaria percutánea) o fibrinólisis. Esto, fomentado por las cada vez más disponibles salas de hemodinamia y centros con capacidad de hacer fibrinolisis en nuestra ciudad ${ }^{34}$. Al finalizar los 90 se recomendó el uso de fibrinolisis en los pacientes con bloqueo de rama izquierda y síntomas anginosos con base en una revisión sistemática realizada por el grupo "Fibrinolytic Therapy Trialists", que basado en una serie de más de 1000 pacientes con sospecha de infarto que recibieron terapia fibrinolítica, encontró una reducción de la mortalidad entre los que presentaron bloqueo de rama al ingreso (25\%), sobre todo si dicho procedimiento se realizaba en las primeras 12 horas de aparición de los síntomas. La gran crítica a dicho trabajo se fundamenta en que en varios de los estudios incluidos no diferenciaron entre bloqueo de rama izquierda y derecha ${ }^{35}$.

Posteriormente, se propusieron algoritmos de manejo para pacientes con bloqueo de rama izquierda y síntomas anginosos, donde por falta de estudios clínicos aleatorizados con criterios bien definidos, se siguió recomendando la fibrinolisis a pesar de la controversia generada por los efectos adversos que pudiesen presentarse en pacientes que no estuvieran presentando un evento coronario agudo. Sin embargo, es de resaltar el beneficio que obtuvieron aquellos pacientes que realmente tenían un bloqueo de rama izquierda como manifestación de un infarto agudo de miocardio ${ }^{27}$.

Por todo lo anterior, la poca evidencia obtenida hasta el momento indica que si el paciente se presenta con síntomas anginosos y bloqueo de rama izquierda en el trazado electrocardiográfico ${ }^{36}$, y además se documenta inestabilidad hemodinámica o clínica de falla cardiaca aguda, debe sospecharse isquemia coronaria como desencadenante de los síntomas, por lo cual este tipo de paciente sería candidato a terapia de reperfusión urgente, según la disponibilidad del centro (revascularización coronaria percutánea o fibrinolisis) ${ }^{8}$.

Si no se presenta ninguno de los dos hallazgos mencionados, el paso a seguir está determinado por la aplicación de los criterios de la doctora Sgarbossa, que en caso de puntuación $>3$, implica que la terapia de reperfusión temprana es beneficiosa ${ }^{31}$. Siguiendo el algoritmo, si las respuestas a los pasos previos es negativa, sigue en su orden la toma de electrocardiogramas seriados, monitoreo cardiaco continuo e incluso biomarcadores cardiacos, $y$ 
según sus resultados, se definirá si se requiere estratificación invasiva o no invasiva, como se presenta en el algoritmo a continuación ${ }^{6,37}$.

\section{¿QUÉ HAY RESPECTO AL INFARTO AGUDO MANIFESTADO COMO BLOQUEO DE RAMA DERECHA?}

Para iniciar, un bloqueo de rama derecha del Haz de His (BRDHH) se define con los siguientes cuatro criterios: $\mathrm{QRS}>120 \mathrm{~ms}$, rsr` o rsR`en derivadas de V1 o $\mathrm{V} 2$, onda $\mathrm{S}$ de mayor duración que la onda $\mathrm{R}$ o $>40 \mathrm{mseg}$ en las derivadas DI y V6, y pico de la onda $\mathrm{R}$ normal en $\mathrm{V} 5$ y V6 $6^{37,38}$.

Clásicamente se ha dicho que para hablar de elevación del segmento ST en el contexto de infarto agudo de miocardio, no hay problema con la presencia de bloqueos fasciculares o BRDHH. Sin embargo, se debe tener precaución al interpretar un electrocardiograma con cambios sugestivos de isquemia en el contexto de $\mathrm{BRDHH}^{39,40}$. La rama derecha del Haz de His recibe suministro sanguíneo de ramas penetrantes septales de la arteria del nodo atrial y atrio ventricular (originarias de la arteria descendente posterior). Aunque el mecanismo fisiopatológico del desarrollo del BRDHH en el contexto de un IAM no ha sido aclarado, pareciera que la oclusión de la arteria descendente posterior podría ser responsable de estos cambios electrocardiográficos ${ }^{41,42}$, sin embargo, debe tenerse en cuenta que hay otras causas de su presencia, entre ellas, el bloqueo puede reflejar un daño preexistente o agudo del sistema de conducción proximal o una alteración estructural del miocardio ventricular. Estos pacientes por lo general se presentan con choque cardiogénico o clínica de falla cardiaca descompensada ${ }^{43-45}$. Típicamente cuando existe un BRDHH se esperan encontrar cambios de repolarización como depresión del segmento ST y ondas $T$ invertidas en las precordiales. En este caso se debería prestar atención a las ondas $\mathrm{T}$ y buscar de acuerdo con la clínica, presencia de isquemia aguda, pues puede significar lesión de la arteria coronaria derecha ${ }^{46,47}$.
Aunque existen limitaciones diagnósticas con el BRDHH como posible equivalente ST, el estudio HERO-2 sí demostró que la presencia de $\mathrm{BRDHH}$ en pacientes infartados aumenta de 3 a 4 veces la mortalidad a 30 días, cuando se compara con IAM en cara anterior sin presentar algún tipo de conducción anormal ${ }^{47-49}$. Por tanto, no se logra establecer una equivalencia total del BRDHH y del infarto agudo de miocardio con elevación del ST, como sí se presenta con el BRIHH y las características mencionadas arriba ${ }^{50}$.

\section{CONCLUSIÓN}

El enfoque clínico del IAM en el contexto de un BRIHH es un reto diagnóstico en urgencias. No hay una clara asociación entre los hallazgos clínicos y cambios del electrocardiograma, pero existen herramientas clínicas (inestabilidad hemodinámica, falla cardiaca, características del dolor) y paraclínicas (Criterios de Sgarbossa en el electrocardiograma, entre otros) que permitirán escoger el mejor manejo definitivo para el paciente.

\section{ALGORITMO DE ABORDAJE}

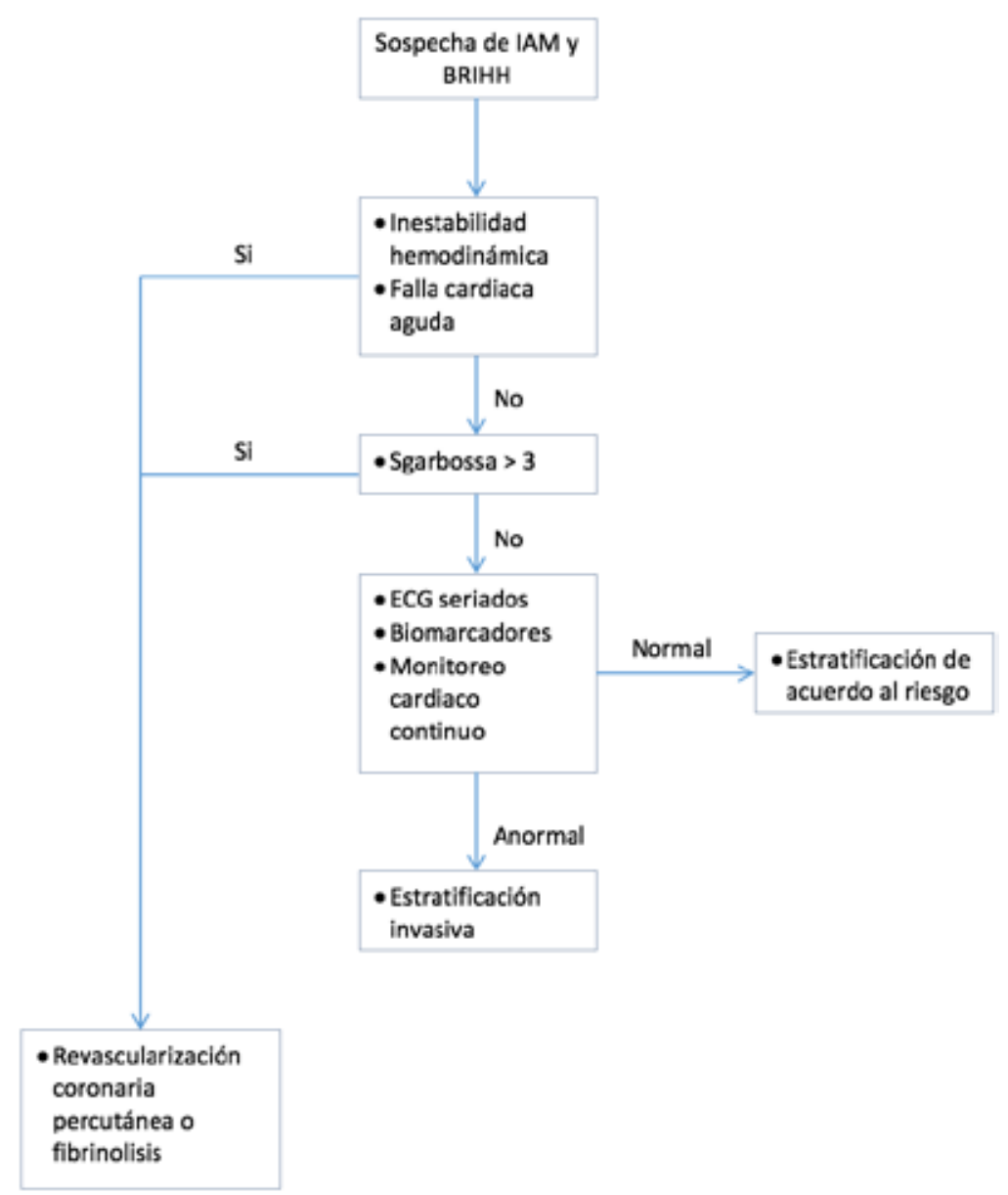




\section{DECLARACIÓN DE CONFLICTO DE INTERESES}

Los autores declaran no tener ningún conflicto de interés.

\section{REFERENCIAS}

1. Morrow DA, Antman EM, Giugliano RP, Cairns R, Charlesworth A, Murphy SA, et al. A simple risk index for rapid initial triage of patients with ST-elevation myocardial infarction: An InTIME II substudy. Lancet 2001; 358(9293):1571-1575.

2. Smith SW, Dodd KW. Letter to the editor regarding «Outcomes in patients with chronicity of left bundle-branch block with possible acute myocardial infarction». Am Heart J. 2011; 162(4):e23.

3. Wellens HJ. Acute myocardial infarction and left bundle-branch block--can we lift the veil? $\mathrm{N}$ Engl J Med 1996; 334(8):528-529.

4. Jain S, Ting HT, Bell M, Bjerke CM, Lennon RJ, Gersh BJ, et al. Utility of left bundle branch block as a diagnostic criterion for acute myocardial infarction. Am J Cardiol 2011; 107(8):1111-1116.

5. Wong C-K, French JK, Aylward PEG, Stewart RAH, Gao W, Armstrong PW, et al. Patients With Prolonged Ischemic Chest Pain and Presumed-New Left Bundle Branch Block Have Heterogeneous Outcomes Depending on the Presence of ST-Segment Changes. J Am Coll Cardiol 2005; 46(1):29-38.

6. Tabas JA, Rodriguez RM, Seligman HK, Goldschlager NF. Electrocardiographic criteria for detecting acute myocardial infarction in patients with left bundle branch block: a meta-analysis. Ann Emerg Med 2008; 52(4):329-336.e1.

7. Madias JE. Left bundle branch block and suspected acute myocardial infarction. J Electrocardiol 2013; 46(1):11-12.

8. Neeland IJ, Kontos MC, de Lemos JA. Evolving considerations in the management of patients with left bundle branch block and suspected myocardial infarction. J Am Coll Cardiol 2012; 60(2):96-105

9. Kontos MC, McQueen RH, Jesse RL, Tatum JL, Ornato JP. Can myocardial infarction be rapidly identified in emergency department patients who have left bundle-branch block? Ann Emerg Med 2001; 37(5):431-438.

10. Larson DM, Menssen KM, Sharkey SW, Duval S, Schwartz RS, Harris J, et al. «False-positive» cardiac catheterization laboratory activation among patients with suspected ST-segment elevation myocardial infarction. JAMA 2007; 298(23):2754-2760.

11. Mehta N, Huang HD, Bandeali S, Wilson JM, Birnbaum Y. Prevalence of acute myocardial infarction in patients with presumably new left bundle-branch block. J Electrocardiol 2012; 45(4):361-367.

12. Coppola G, Carità P, Corrado E, Borrelli A, Rotolo A, Guglielmo M, et al. ST segment elevations: Always a marker of acute myocardial infarction? Indian Heart J 2013; 65(4):412-423.

13. Larson DM, Menssen KM, Sharkey SW, Duval S, Schwartz RS, Harris J, et al. «False-positive» cardiac catheterization laboratory activation among patients with suspected ST-segment elevation myocardial infarction. JAMA 2007; 298(23):2754-2760.

14. Sgarbossa E. Electrocardiographic diagnosis of acute myocardial infarction in the presence of left bundle-branch block: Can we "treat all" patients? Ann Emerg Med 2002; 39(1):97.

15. Sgarbossa EB, Pinski SL, Barbagelata A, Underwood DA, Gates KB, Topol EJ, et al. Electrocardiographic diagnosis of evolving acute myocardial infarction in the presence of left bundle-branch block. GUSTO-1 (Global Utilization of Streptokinase and Tissue Plasminogen Activator for Occluded Coronary Arteries) Investigators. N Engl J Med 1996; 334(8):481-487.

16. Al-Faleh H, Fu Y, Wagner G, Goodman S, Sgarbossa E, Granger C, et al. Unraveling the spectrum of left bundle branch block in acute myocardial infarction: insights from the Assessment of the Safety and Efficacy of a New Thrombolytic (ASSENT 2 and 3) trials. Am Heart J 2006; 151(1):1015.

17. Sgarbossa EB. Recent advances in the electrocardiographic diagnosis of myocardial infarction: left bundle branch block and pacing. Pacing Clin Electrophysiol 1996; 19(9):1370-1379.

18. Edhouse JA, Sakr M, Angus J, Morris FP. Suspected myocardial infarction and left bundle branch block: Electrocardiographic indicators of acute ischaemia. J Accid Emerg Med 1999; 16(5):331335.

19. Lopes RD, Siha H, Fu Y, Mehta RH, Patel MR, Armstrong PW, et al. Diagnosing acute myocardial infarction in patients with left bundle branch block. Am J Cardiol 2011; 108(6):782-788.

20. Hindman MC, Wagner GS, JaRo M, Atkins JM, Scheinman MM, DeSanctis RW, et al. The clinical significance of bundle branch block complicating acute myocardial infarction. 1. Clinical characteristics, hospital mortality, and one-year follow-up. Circulation 1978; 58(4):679-688.

21. Col JJ, Weinberg SL. The incidence and mortality of intraventricular conduction defects in acute myocardial infarction. Am J Cardiol 1972; 29(3):344-350. 
22. Fesmire FM. ECG diagnosis of acute myocardial infarction in the presence of left bundle-branch block in patients undergoing continuous ECG monitoring. Ann Emerg Med 1995; 26(1):69-82.

23. Smith SW, Heegaard W, Bachour FA, Brady WJ. Acute myocardial infarction with left bundlebranch block: disproportional anterior ST elevation due to right ventricular myocardial infarction in the presence of left bundle-branch block. Am J Emerg Med 2008; 26(3):342-347.

24. Shlipak MG, Lyons WL, Go AS, Chou TM, Evans GT, Browner WS. Should the electrocardiogram be used to guide therapy for patients with left bundle-branch block and suspected myocardial infarction? JAMA 1999; 281(8):714-719.

25. Chapman MG, Pearce ML. Electrocardiographic diagnosis of myocardial infarction in the presence of left bundle-branch block. Circulation 1957; 16(4):558-571.

26. Madias JE. Left bundle branch block and suspected acute myocardial infarction. J Electrocardiol 2013; 46(1):11-12.

27. Gallagher EJ. Which patients with suspected myocardial ischemia and left bundle-branch block should receive thrombolytic agents? Ann Emerg Med 2001; 37(5):439-444.

28. Clinical Policy: Critical Issues in the Evaluation and Management of Adult Patients Presenting With Suspected Acute Myocardial Infarction or Unstable Angina. Ann Emerg Med 2000; 35(5):521544.

29. Li SF, Walden PL, Marcilla O, Gallagher EJ. Electrocardiographic diagnosis of myocardial infarction in patients with left bundle branch block. Ann Emerg Med 2000; 36(6):561-565.

30. Bansilal S, Aneja A, Mathew V, Reeder GS, Smars PA, Lennon RJ, et al. Long-Term Cardiovascular Outcomes In Patients With Angina Pectoris Presenting With Bundle Branch Block. Am J Cardiol 2011; 107(11):1565-1570.

31. Cai Q, Mehta N, Sgarbossa EB, Pinski SL, Wagner GS, Califf RM, et al. The left bundle-branch block puzzle in the 2013 ST-elevation myocardial infarction guideline: from falsely declaring emergency to denying reperfusion in a high-risk population. Are the Sgarbossa Criteria ready for prime time? Am Heart J 2013; 166(3):409-413.

32. Barron HV, Bowlby LJ, Breen T, Rogers WJ, Canto JG, Zhang Y, et al. Use of reperfusion therapy for acute myocardial infarction in the United States: data from the National Registry of Myocardial Infarction 2. Circulation 1998; 97(12):1150-1156.

33. Fibrinolytic Therapy Trialists' (FTT) Collaborative Group. Indications for fibrinolytic therapy in suspected acute myocardial infarction: collaborative overview of early mortality and major morbidity results from all randomised trials of more than 1000 patients. Lancet 1994; 343(8893):311-322.

34. Chang AM, Shofer FS, Tabas JA, Magid DJ, McCusker CM, Hollander JE. Lack of association between left bundle-branch block and acute myocardial infarction in symptomatic ED patients. Am J Emerg Med 2009; 27(8):916-921.

35. Indications for fibrinolytic therapy in suspected acute myocardial infarction. Lancet 1994; 343(8893):311.

36. Madias JE, Sinha A, Ashtiani R, Agarwal H, Win M, Narayan VK. A critique of the new ST-segment criteria for the diagnosis of acute myocardial infarction in patients with left bundle-branch block. Clin Cardiol 2001; 24(10):652-655.

37. Hollander JE, Blomkalns AL, Brogan GX, Diercks DB, Field JM, Garvey JL, et al. Standardized reporting guidelines for studies evaluating risk stratification of emergency department patients with potential acute coronary syndromes. Ann Emerg Med 2004; 44(6):589-598.

38. Surawicz B, Childers R, Deal BJ, Gettes LS, Bailey JJ, Gorgels A, et al. AHA/ACCF/HRS recommendations for the standardization and interpretation of the electrocardiogram: Part III: Intraventricular conduction disturbances: A scientific statement from the American Heart Association Electrocardiography and Arrhythmias Committee, Council on Clinical Cardiology; the American College of Cardiology Foundation; and the Heart Rhythm Society. Endorsed by the International Society for Computerized Electrocardiology. J Am Coll Cardiol 2009; 53(11):976981.

39. Vogler J, Breithardt G, Eckardt L. Bradyarrhythmias and conduction blocks. Rev Espanola Cardiol Engl Ed 2012; 65(7):656-667.

40. Gussak I, Wright RS, Bjerregaard P, Chaitman BR, Zhou SH, Hammill SC, et al. False-negative and false-positive ECG diagnoses of $\mathrm{Q}$ wave myocardial infarction in the presence of right bundlebranch block. Cardiology 2000; 94(3):165-172.

41. Celik M, Iyisoy A, Celik T. Intermittent right bundle branch block development in a patent with acute inferior myocardial infarction. Int J Cardiol 2011; 150(3):e121-123.

42. Marques-Vidal P, Rodondi N, Bochud M, Chiolero A, Pécoud A, Hayoz D, et al. Predictive accuracy of original and recalibrated Framingham risk score in the Swiss population. Int J Cardiol 2009; 133(3):346-353.

43. Berning J, Steensgaard-Hansen F. Early estimation of risk by echocardiographic determination of wall motion index in an unselected population with acute myocardial infarction. Am J Cardiol 1990; 65(9):567-576.

44. Stenestrand U, Tabrizi F, Lindbäck J, Englund A, Rosenqvist M, Wallentin L. Comorbidity and myocardial dysfunction are the main explanations for the higher 1-year mortality in acute myocardial infarction with left bundle-branch block. Circulation 2004; 110(14):1896-1902. 
45. Lewinter C, Torp-Pedersen C, Cleland JGF, Køber L. Right and left bundle branch block as predictors of long-term mortality following myocardial infarction. Eur J Heart Fail 2011; 13(12):1349-1354.

46. Wagner GS, Macfarlane $P$, Wellens $H$, Josephson $M$, Gorgels $A$, Mirvis DM, et al. AHA/ACCF/ HRS recommendations for the standardization and interpretation of the electrocardiogram: part VI: Acute ischemia/infarction: A scientific statement from the American Heart Association Electrocardiography and Arrhythmias Committee, Council on Clinical Cardiology; the American College of Cardiology Foundation; and the Heart Rhythm Society. Endorsed by the International Society for Computerized Electrocardiology. J Am Coll Cardiol 2009; 53(11):1003-1011.

47. Cooper BL, Khosla AJ, Voronin BW. Myocardial infarction in the presence of a right bundle branch block. J Emerg Med 2016; 50(5):778-779.

48. Pozen JM, Mankad AK, Owens JT, Jovin IS. New right bundle branch block as a criterion for emergent coronary angiography. North Am J Med Sci 2015; 7(12):569-571.

49. Wong C-K, Stewart RAH, Gao W, French JK, Raffel C, White HD. Prognostic differences between different types of bundle branch block during the early phase of acute myocardial infarction: insights from the Hirulog and Early Reperfusion or Occlusion (HERO)-2 trial. Eur Heart J 2006; 27(1):21-28.

50. Ibanez B, James S, Agewall S, Antunes MJ, Bucciarelli-Ducci C, Bueno H, et al. 2017 ESC Guidelines for the management of acute myocardial infarction in patients presenting with STsegment elevationThe Task Force for the management of acute myocardial infarction in patients presenting with ST-segment elevation of the European Society of Cardiology (ESC). Eur Heart J 2018; 39(2):119-177. 